\title{
DEMOCARE: A mixed kinship microsimulation and Agent-Based models for studying family supply of time for care of elderly people with disabilities ${ }^{1}$
}

Daniel Devolder ${ }^{2}$

Jeroen Spijker

Pilar Zueras

Centre d’Estudis Demogràfics, Universitat Autònoma de Barcelona, Bellaterra, Spain

\section{Abstract}

This paper presents a mixed model for studying the amount of time of care available from family members of elderly people with disabilities that affect their ability to carry out (instrumental) activities of daily living. This model starts with data obtained from a classical microsimulation model of kinship which provides descending genealogies of elderly persons, based on demographic parameters specifics to the population studied. These genealogies are then fed to an Agent-Based model which follow these elderly persons and their kin for age 50 until their death in order to study the balance between the demand for care of disabled persons and the supply of care by their kin. This supply depends on the situation of these kin, typically of their labour status as well as the eventual needs for care of their own families, in particular if they have young children of their own. The paper reviews the data requirements and methodology for the construction of microsimulation models of kinship and presents our own implementation. It also details the specifications of the agent-based part of the overall model. Finally, we present some results using Spanish data.

\footnotetext{
${ }^{1}$ This work was supported by the Spanish Ministry of Science, Innovation and Universities under the 'Ramón y Cajal' programme (JS, RYC-2013-14851) and the 'Juan de la Cierva' programme (PZ, FJCI-2015-27107), and forms part of the R\&D project 'Will future elderly have any relative available to care for them? A study based on a mixed micro-simulation/ABM model' (CSO2017-89721-R) co-directed by DD and JS and financed by the same ministry; and the Catalan government under the CERCA programme.

${ }^{2}$ Corresponding author: ddevolder@ced.uab.es
} 


\section{Introduction}

The purpose of this article is to explain in more detail the data requirements and methodology for the construction of a recently developed mixed microsimulation-agent-based model (ABM) we called Democare. It allows to comprehensively study the demand and supply of informal care for elderly persons in situations of dependence, based on their available network of relatives. The model was recently briefly described and used to study the impact of demographic change in the balance between formal and informal old-age care in Spain (Spijker, Devolder, \& Zueras, 2020) However, the parameter specifications and functions required in the microsimulation kinship and $A B M$ models were suppressed for reasons of space. Moreover, the current version also has several new additions, including the possibility for an individual to separate from their partner.

Democare uses two different simulation techniques. First, a microsimulation model provides kinship networks of representative individuals, called "egos", based on the demographic characteristics of each cohort and, second, an Agent Based Model (ABM) simulates the life of individuals belonging to these networks until the death of egos, thus making it possible to estimate the care demand of these egos that could be satisfied by their close kin network (partner and children). Likewise, the ABM calculates the proportion of the care demand that cannot be assumed by informal family resources. This corresponds to the part that needs to be externalized, presumably to the formal sphere. ${ }^{3}$

In this paper, first we review in detail the methodology used for the construction of microsimulation models of kinship, and compare existing models with the one we build and applied in DemoCare. Then we describe the ABM module and how it allows us to combine a great detail of demographic information provided by the microsimulation model and socioeconomic and health data obtained from a health survey.

\footnotetext{
${ }^{3}$ For reconstruction of the kinship network, the Pascal language is used, as described in Devolder (2002, 2004). Regarding the ABM used to estimate the supply and demand of hours of care throughout the ego's life the NetLogo software is used, a programming environment especially designed for the development of ABM and simulation of natural and social phenomena. In Calduch et al. (2017) a more detailed description of the DemoCare model is given but we envisage making both programmes available to other researchers so that DemoCare can be applied to other countries that have the necessary data.
} 


\section{Background}

Over the last couple of decades, there has been a resurging interest in computer simulation and ABM in the social sciences (Billari \& Prskawetz, 2003; Silverman, Bijak, Noble, Cao, \& Hilton, 2014). It has been applied as a tool to model residential segregation (e.g. Bruch, 2014; Clark and Fossett, 2008; Zhang, 2011), migration (e.g. Heiland, 2003; Kniveton et al., 2011; Willekens, 2012), partnership, family formation and divorce (e.g. Aparicio Diaz et al., 2011; Bijak et al., 2013; Billari et al., 2007; Grow et al., 2017), and household dynamics (e.g. Geard et al., 2013), but perhaps less to elderly care. Spielauer (2007) reviewed 27 dynamic microsimulation models applied to health studies, among which LifePaths, developed at Statistics Canada (Carrière, Keefe, Légaré, Lin, \& Rowe, 2007), and APPSIM, at the University of Canberra (Nepal, Brown, Kelly, \& Percival, 2011), are close to our model, inasmuch as they were applied to the same subject, the balance between the demand and supply of care for oldage dependent persons, and operated at the family level, taking into account as much variability as possible instead of working on aggregates (see also Gaymu et al. (2010) for a similar application using a multistate demographic model). Ma, Shen, \& Nguyen (2016) developed an ABM to help local governments in Japan review their planning strategy of developing elderly day care centres, while Noble et al. (2012) studied the implications of household formation and other demographic processes in the supply of, and demand for social care in the UK.

Compared with other applications of microsimulation and ABM modelling, Democare stands out as it simulates in more detail the supply of care that an elderly dependent person can potentially receive from her or his kinship network. For example, Carrière, Keefe, Légaré, Lin, \& Rowe (2007); Gaymu, Ekamper, \& Beets (2007) considered close family composition (whether or not with a partner and surviving children) in their care models but, unlike Democare, did not take into account interactions within the kinship network and factors than can constrain the potential availability of kin as carers.

By contrast, our model starts with the close kin network of simulated individuals, from the extreme situation of people without any close relative to those who have a partner and many children and grandchildren, replicating the variability of real kinship networks as much as possible. The key difference with previous models is that Democare also takes into account 
other factors that may constrain the supply of care from kin, namely labour force participation and own health status but, importantly, the demand for care from all the other kin in the network as well. Our demand-supply care model is the first of its kind for the Spanish context, and the lifecycle perspective for this kind of model is, as far as we know, also new. Noble et al. (2012), for instance, developed a similar simulation model, but at the household rather than kinship level and without considering personal characteristics of kin that constrain supply. Their results are expressed at the population level, using a period perspective when, with Democare, they are presented using a cohort approach at an individual level.

\section{The microsimulation module of the model}

In this section we present the KINFERT kinship simulation module used in Democare. This is a microsimulation model of kinship that we first compare with similar existing models before detailing their internal structure. The presentation starts with an examination of the KINFERT fertility module (which corresponds to the FERT part of the acronym), before proceeding to the analysis of the kinship simulation algorithms themselves (the KIN part of the acronym).

\subsection{Fertility microsimulation module: complete specification for the parameters and functions used in the microsimulation model}

We simulate the reproductive live of a set of women, from birth until 59 years of age. Our model is based on the following set of hypotheses, in which we take into account the biological dimension of fertility (parameters a to h) as well as those that depend on social or individual choices (parameters i to $n$ ).

a. Progression of sterility with age: we followed the procedure used by Léridon (2008) and calibrated our model modifying the sterility function until our results fitted the cumulated proportions of last birth by woman's age, as collected by Louis Henry for Ancient France. Our final sterility distribution by age differs from Léridon (2008), due to internal differences in the microsimulation model (right curves in the following graph). We checked the results of our model computing childlessness levels at age 50 , for groups of women who marry at the same age, and we compared the proportion obtained with observed one taken from the South 
African Population Census of 1921, for white women, which is one of the best datasets available for non-controlling populations (left curves).

Figure 1. Sterility by woman's age in the model compared to Leridon's one and simulated childlessness by woman's age at marriage compared to South African 1921 Census data

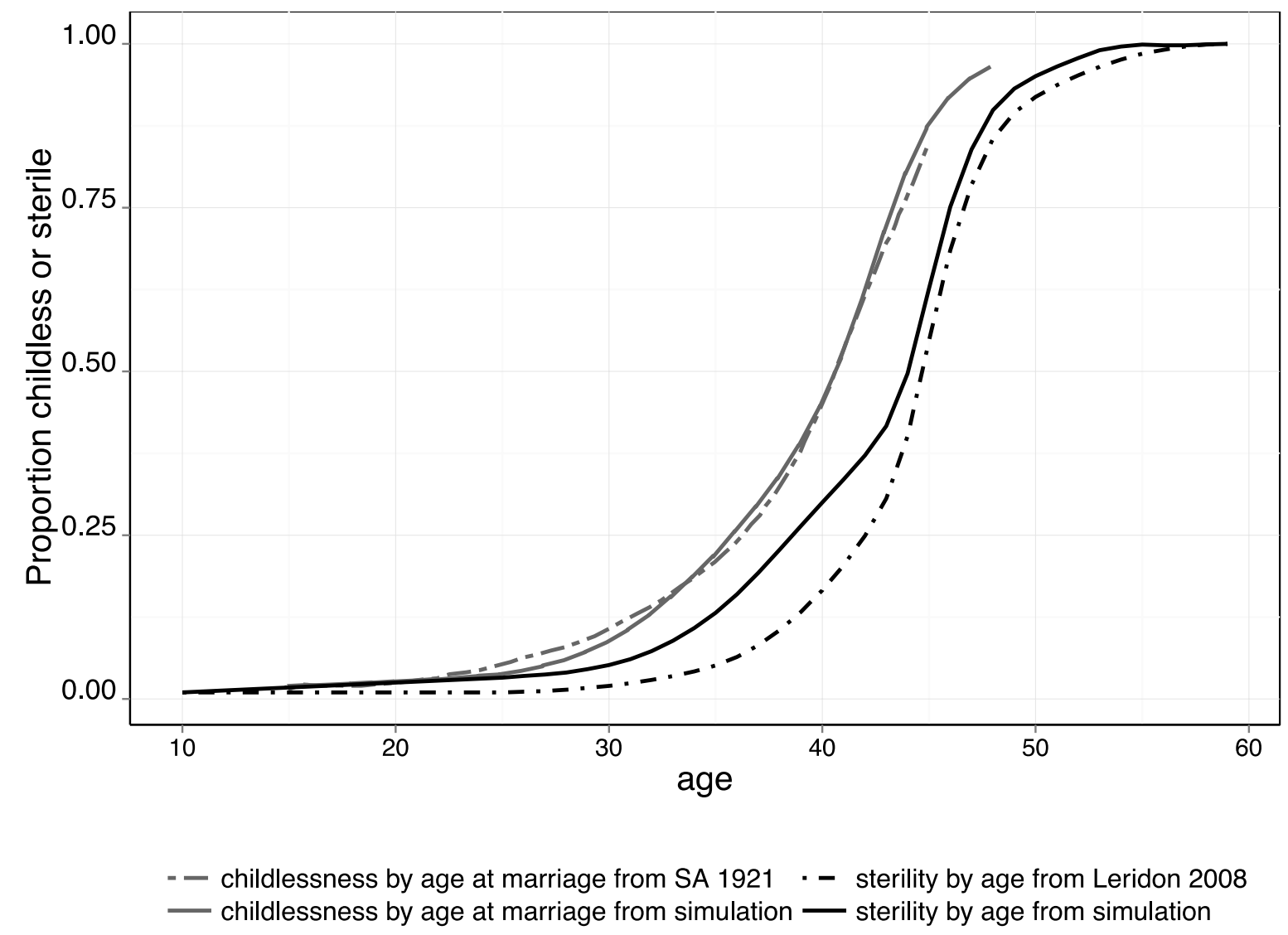

b. Evolution of the risk of intrauterine mortality with the duration of the pregnancy: model derived from data in Léridon (2004) fitted with a third-degree polynomial:

$m(d)=0.0509152+0.0093173 d-0.0004664 d^{2}+0.0000086667 d^{3}$

Where $d$ is the duration in menstrual cycles.

c. Risk of intrauterine mortality during pregnancy: Barrett (1978) formula:

$\operatorname{mi}(d)=0.11(0.55)^{d-2}$ with $2 \leq d \leq 8$

Where $d$ is the duration in menstrual cycles. 
d. Risk of late foetal mortality with age: Barrett (1978) formula:

$m f(x)=0.24+0.005(x-30)$

where $x$ is age in years.

e. Duration of pregnancy: for a live birth, same value of 10 menstrual cycles (of 28 days each) for all women; 9 menstrual cycles for a stillbirth; for other foetal deaths, the duration corresponds to the value determined by Barrett's formula in point c.

f. Heterogeneity of fecundability distributed following a normal function. Between age 20 and 35 , the mean is equal to 0.23 and the standard deviation to 0.12 . Each woman is assigned a relative fecundability level after a random trial on this normal function, and this relative level is held constant for all her fecund life.

g. Mean fecundability with age, same than Léridon (2004): a linear function from age 15 until 20 years, with a variation from 0 to 0.23 ; then a constant value up to age 35; finally, the fecundability level falls linearly until the end of the fecund life (different for each woman, equal to their age at permanent sterility).

h. Distribution of the risk of temporary sterility after a childbirth (post-partum amenorrhea): Lesthaeghe \& Page (1980) model, with values for the model parameters so as to obtain a very short duration, with a median duration of around 4 cycles (alpha $=-1.2$ et beta $=1$ ).

i. Fertility control (use of efficient contraception) implemented as ex ante parity progression ratios inferior to 1 , based on desired fertility levels (table A2). That means stopping only. Spacing is implemented only for the first interval, between first union formation and first pregnancy: see point $\mathrm{n}$.

j. First partnership formation risks for women: Coale \& McNeil (1972) model, using the reparametrization by Rodriguez \& Trussel (1980). Standard deviation varies with mean age $\mu$ using the formula:

$$
\sigma=\sqrt{\frac{45}{1+500 e^{\left(-\frac{\mu}{2}+5\right)}}}
$$

k. First partnership formation risks for men: the mean difference in age at first partnership between men and women is set at 2 years. We use again Rodriguez \& Trussel (1980) model, 
but computing a distribution for men for each age at first partnership formation of women, in the age interval $10-59$ years.

I. Higher order union formation: the final proportion of separated or widowed women is set to a fixed value, as indicated in the text. The distribution of duration between the time the women separates and the next union is the same as for the process of entry in first union, but using as starting point the age at which the proportion of women in union is superior or equal to $2 \%$ in the previous distribution.

$m$. Divorce or union dissolution risk: the distribution of risk in function of the union duration follows a generalized log-logistic model (Brüederl \& Diekmann, 1995). The parameters values are the followings: alpha $=1.7$, beta $=0.01$ and lambda $=0.015$. The final proportion of union dissolution is reached 30 years after the start of the union. The risk varies with the number of living children, following relative risks values estimated by Toulemon (1995) for French data and (Andersson, 1997) for Sweden data. The values of the relative risks multipliers, the reference situation been childless, are the following (intermediate values are interpolated):

Table 1: Relative risks multipliers of separation associated with the presence of children under 25 years of age.

\begin{tabular}{c|c|c}
\hline $\begin{array}{c}\text { Duration since the childbirth } \\
\text { (in menstrual cycles) }\end{array}$ & First birth & Second and higher birth orders \\
\hline-11 & 1.0 & Current value \\
-6 & 0.15 & 0.075 \\
7 & 0.05 & 0.025 \\
20 & 0.225 & 0.1125 \\
52 & 0.45 & 0.175 \\
65 & 0.5 & 0.1925 \\
156 & 0.7 & 0.3 \\
260 & 0.8 & 0.4 \\
325 & 1.0 & 1.0 \\
\hline
\end{tabular}

The microsimulation model is iterated until the resultant proportion of separation is the same than the one entered as input. 
$\mathrm{n}$. Distribution of waiting time between the union formation and the time when couples no longer use contraceptive means follows a Poisson law:

$p(m)=e^{-\lambda} \frac{\lambda^{m}}{m !}$

Where $m$ is the menstrual cycle starting at which contraception is no longer used, and $m=0$ corresponds to the start of the union. The parameter $\lambda$ is a mean number of menstrual cycles, which is equal to the value in column 3 of table A5, less 6 months (which is the mean value for a non-contraceptive population). The use of contraception at the start of the union before the first conception corresponds only to a proportion of couples, variable between countries, and for the rest of couples there is no 'waiting time'.

o. The mortality level is the same for all countries and corresponds to the "West" model life table level 25 of Coale, Demeny, \& Vaughan (1983). Mortality here is for male only, for computing the risk of widowing.

\subsection{Kinship microsimulation models}

The first micro-simulation models of kinship appeared in the 1960sthat were developed by anthropologists and historians, often in connection with the pioneering works of Orcutt on socio-economic microsimulation models (Hammel, 1990). The questions anthropologists and historians were asking at the time were about the degree of endogamy in marriages, the availability of cousins, the effects of the prohibition of incest or first cousin marriages on demographic viability, the effect of the density of the kinship network on household structure.

In this paper we will focus on how these programmes work and how well they perform in terms of the obtention of individual probabilities of the availability of relatives who can be considered potential caregivers. There are different approaches to the simulation of kinship networks. When one wants to obtain aggregated results, the use of microsimulation is not necessarily the best solution. For example, one can calculate the average risks or expected number of having a given type of living relative, for example parents, grandparents, cousins, children, etc., from analytical formulas such as those of Goodman, Keyfitz, \& Pullum (1974) developed and detailed also in Keyfitz \& Caswell (2005). But these formulas are not practical 
for calculating the distributions of the number of relatives, and for example their variance. Moreover, it should be noted that they include multiple integrals whose dimension increases directly with the degree of relatedness. In fact, the use of numerical methods, based on Monte Carlo algorithms, will be necessary to obtain results, which in fine will be equivalent to a microsimulation.

Another alternative is the use of macrosimulation models, based on the multi-state methodology (the best-known model is LIPRO, which is very general, but has been applied mainly to household projections: van Imhoff, 2005). The main problem with these models is their complexity if many situations are to be taken into account. For example, the interaction between union and fertility already leads to a high number of states to be taken into account, especially if, for example, separations and second unions come into play, or conceptions prior to unions. The advantage of microsimulation in this context is simplification, both in comparison with analytical models and with macrosimulations. An additional advantage is the integration of time at a finer level, even in continuous time. For practical reasons, analytical or macrosimulation models are estimated for ages and periods that are usually annual or multiannual. This can create problems when competing and incompatible events occur in the same time period, for example if a union or conception are simulated to occur close to the death of one of the partners. Another important aspect is that it is much easier to incorporate individual or family heterogeneity into a microsimulation. Thus, as discussed in the previous sub-section, there are differences between individuals which may be biological, such as the degree of fertility for women, or linked to genetic inheritance at the family level, or even social status, such as the level of education which conditions behaviour and which is also often correlated between parents and children. In a microsimulation, risk modelling can take into account this heterogeneity throughout the life cycle, which is impossible in a macrosimulation and difficult for an analytical model. The consequence is that a microsimulation will in general allow for a greater diversity of situations at the individual level. Finally, one advantage that may be decisive is precisely the ability to offer the creation of virtual populations at the individual level, and thus to be able to have the detailed structure of the kinship of a group of people that is more or less important according to needs. It is precisely this point that was important for the present modelling, since the kinship model had to provide individual data that are used to feed the agent-based model. 


\subsubsection{A taxonomy of microsimulation models of kinship}

These models are differentiated by the following characteristics:

a) Population- or individual- (ego-) based. Most of the existing socio-demographic microsimulation models use a complete population as a basis for the simulation. This population is a list of individuals, usually living with others in a household. This list is obtained from a census, a survey or a population register, or can be a synthetic population created ad hoc by the model. This means that the simulation starts with individuals of different ages, and that the initial information on kinship is usually limited to the co-residents. On the contrary other models starts from individuals, usually denominated 'ego', and the simulation is then usually cohort based, following a set of egos born the same year. Then for an ego-based simulation, obtaining results at the population level requires the simulation of egos of successive cohorts that make up a complete population.

b) Open, partially- or fully-closed. Some models are open in the sense that when a union is simulated, the model needs to generate a new individual who do not pertain to the existing pool present in the simulation. On the contrary others models are fully-closed, as kin by alliance form part of the population simulated in previous steps and biological kin generated by the model increase the population count in the present step. A few population models are partially-closed as only biological kin contribute to demographic increase, and kin by alliance does not form part of population sets simulated in previous steps. Ego-based simulation are by force open in the sense that no ego shares kin with other egos. On the contrary partially or fully closed models are always population-based. A big advantage of fully-closed models is that, as the in-law kin selected already exists in the population, her or his kinship network is already created. On the contrary in an open or partially-closed model, the kin who enter by union are created ex nihilo, and we have no information on their kinship (the kinship network can be created if needed, but this calls for an extra step).

c) Forward (projective type) models for which all the simulated kin are descendants of the original individuals, or backward-forward models which also go backward in time and simulate the availability of ancestors, especially the parents, aunts and uncles, as well as the grandparents or even the great-grandparents. Backward models are especially useful as they allow simulating directly the lateral part of the genealogy, and for example first cousins are reconstructed after a backward simulation has reached grandparents' level: the simulation 
then can proceed downward starting from aunts and uncles of egos whose children are their first cousins. By definition population-based models can be only of the projective or forward type and backward simulations are possible only in ego-based simulations.

Interpretations of the results obtained by the models also differ. Ego-based models usually are of a probabilistic kind and give expected numbers of kin of any type born or alive at each age of ego, as well as the distribution of these numbers. On the contrary with populationbased models the interest is more on changes in composition over time, by kin type or household characteristics.

\subsubsection{Main microsimulation models of kinship}

There are relatively few 'pure' kinship microsimulation models. In fact only SOCSIM and CAMSIM, presented in detail in the next sections, appear in most reviews of the literature (for example Willekens, 2010; Zagheni, 2015). On the other hand, comparable socio-demoeconomic microsimulation models, in the sense that their demographic module is very similar and serves the same purpose of reconstructing elementary kinship, are much more abundant, and increasingly used for all types of applications, economic, health, or purely demographic. Most of these models actually have an algorithmic structure very similar to SOCSIM: they are all based on a specific initial population, using observational data, and the analysis of its structural effects and their social and economic consequences is carried out by a projection of the kinship of individuals into the future, usually coupled with co-residency rules.

\subsubsection{Population-based models}

\subsection{SOCSIM and others fully-closed projective models}

SOCSIM (Social Simulation) is the oldest "pure" microsimulation model of kinship and probably the most used. The first version was published in the 1970s (Wachter, Hammel, \& Laslett, 1978) and it has continued to evolve since then. The source code has been available since the first version, by requesting it by email from the authors. The model originally used the FORTRAN language, while the second version was written in Pascal (Hammel, 1990), while more recent versions use the $C$ language (Mason, 2016). It is a development of earlier anthropological simulation of small populations (Gilbert \& Hammel, 1966) with relatively little 
openness to the outside world and for which the problem of the rules of the matrimonial market arises, for example the acceptance or rejection of unions between first cousins, in the context of demographic sustainability. This partly explains why SOCSIM is from the outset a model based on a closed population type, i.e. for which the biological kin or in-laws of individuals are fully included in the simulated population. The results of the simulation are strongly dependent on the size and structure of the initial population, which also condition the functioning of the mate matching algorithm. The reconstruction of kinship genealogies requires a projection over several generations, and a general probabilistic interpretation of the results, which depends only on the level of fertility and mortality, is not immediate. This is only possible if the simulation is continued over several generations so that the structural effects of the initial population disappear (a so-called ergodic process), or if this initial population has, for example, a stable structure from the outset, and that its size is sufficiently large for the matrimonial market not to cause rationing effects, conditions which in fact go against the logic of the model. The fertility model in SOCSIM is very simplified, based on ageparity-specific month-to-month rates common to all individuals. The effect of individual heterogeneity due to permanent biological differences in fertility was added in more recent versions, which allows for increased variability in family size, but this is done by a simple multiplier of the age-specific rates. On the other hand, the model does not take directly into account heterogeneity linked to the progression of sterility with age, but only indirectly, through the profile of the age-specific rates.

The functioning of SOCSIM is illustrated in the following figure. We start with a population by age and sex at time $t$, with possible information on marital status (represented by a union link) and on offspring (represented by a link between mothers and the eldest child of the sib group). Usually, this information is not exhaustive and is limited to co-resident relatives in a household. The simulation then consists of extending the descending genealogies month by month. For example, individuals 1 and 2 are siblings of a child already born at time $t$ and 3 and 4 are descendants of a couple present in the initial population, without co-resident children. The descending genealogies can then be extended to the grandchildren (case of individuals 6 and 7, who are also first cousins) and continued over several generations in order to obtain a complete genealogy for a simulated individual (and obtain, for example, his grandparents, parents, cousins, children and grandchildren). This is what SOCSIM does by 
continuing the simulation for example up to 200 years after time $t$, so that these complete genealogies can be representative of the conditions of the demographic regime and no longer depend on the composition of the population at time $t$. On the other hand, if the interest is in discovering the importance of initial conditions on demographic and family dynamics, the projection horizon can be much shorter.

In fact, for the majority of socio-economic microsimulation models that are algorithmically similar to SOCSIM, the interest is much less in reconstructing individual genealogies than in exploring the effects of the variability of the family situation of existing households at time $t$. Thus a model such as Orcutt's DYNASIM, which is the precursor of the dynamic microsimulation models that have proliferated since the 1990s (see Li \& O'Donoghue (2012) or Morand, Toulemon, Pennec, Baggio, \& Billari (2010) for an in-depth review of these models), the projection horizon is of about 60 years, sufficient for most of the households in the original population to have disappeared.

It is interesting to observe that most of the population-based models similar to DYNASIM are fully closed and include a mate-matching algorithm, which is often the most complex part of the model (see for example Bacon \& Pennec, 2007). The reason for this is that the results of socio-economic population-based microsimulation models are normally aggregated and compared to socio-economic data at the macro level, and in that sense should be consistent with results obtained by a traditional population projection (in that sense MicMac is the most refined model of this kind, as its methodology is based on an automatic alignment of results at the micro and macro levels: Willekens (2005).

Figure 2. SOCSIM algorithm: closed population-based projective microsimulation model
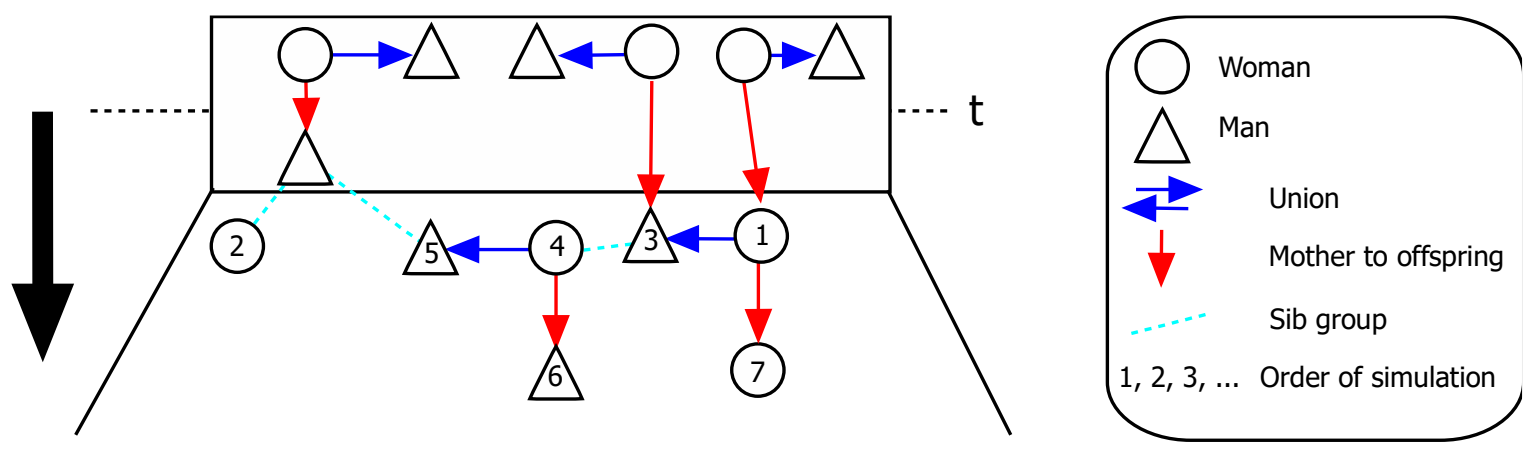

SOCSIM 


\subsection{Partially-closed projective models}

As mentioned earlier, most models based on the projection of an initial population over time are fully closed. However, some are only partially closed due to the fact that they do not include a mate-matching algorithm, and thus the simulation of a union will lead to the appearance of a new person, who did not previously exist in the simulated population, a kind of hidden immigration. This means that the simulation will no longer give consistent results at the macro level, but still valid at the individual or household level. This approach is not very common, as for example only 8 of the 52 dynamic microsimulation models cited in (Li \& O'Donoghue, 2013) are of this type.

Figure 3 presents genealogies created by a partially-closed model. The first obvious difference with a fully-closed one is that when a union is simulated by the model, new individuals are created ex-nihilo, outside of the existing pool. For example, individuals 4 and 8 appears from the outside, and as a consequence do not have their proper kinship network. In that sense they appear "out of the blue", at the age simulated for the union and without a previous simulated life. Another consequence is that the coherence between the micro and the macro level disappears in the sense that the population count at the micro level deviates instantly from what we would obtain from a macro-projection using the same basic hypothesis of future fertility and mortality. Another importance difference, at the algorithmic level, is that in a partially-closed model, the forward or descendant simulation of kinship operates at the household level: each genealogy is completed, before turning to another household of the population at time $t$ : as can be seen from the figure the order of simulation of individual runs from 1 to 5 for the first household and then from 6 to 10 for the second one. On the contrary, a closed model like SOCSIM scan all the individuals of the pool each month of the simulation and the order of simulation of individuals depends on the date of events. 
Figure 3. Algorithm of a partially-closed projective-type microsimulation model
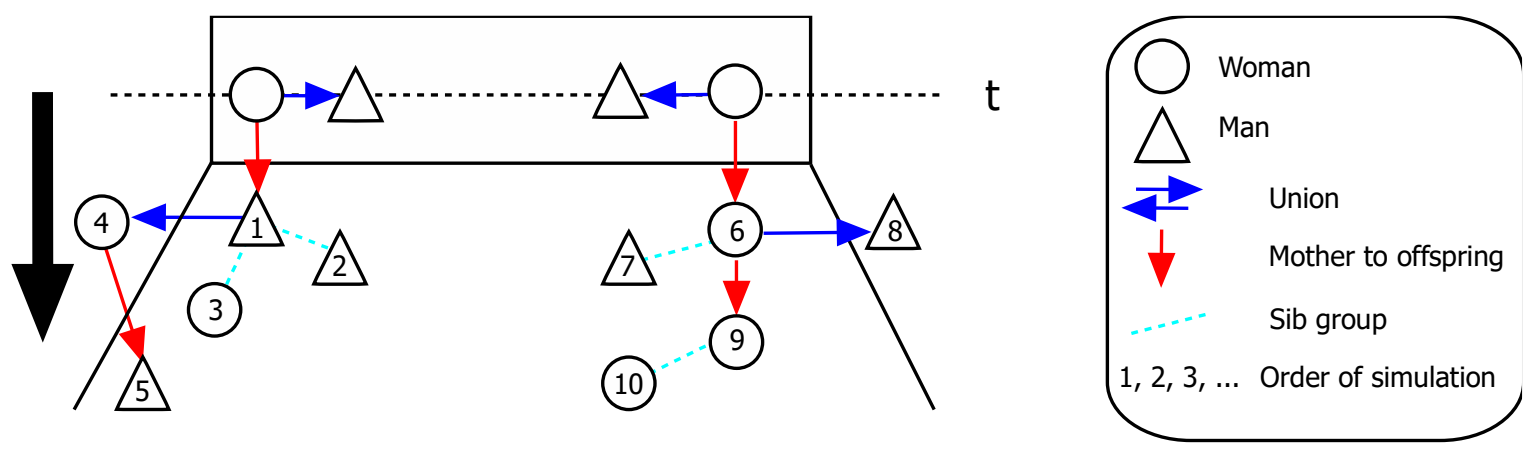

Partially-closed

\subsubsection{Ego-based models: looking for the forebears}

In contrast to population-based models, "ego-based" models use from the outset a pure probabilistic approach: if one takes a person at random from a population, one will be interested in knowing the probability that she or he had children and their expected numbers, that his or her mother or father is alive, etc. In fact, ego-based models seem to be practical developments of the analytical methods presented in Goodman et al. (1974) who generalized Lotka's (1931) approach for computing orphanhood probabilities and extended it to the rest of relatives. But in fact, the later limited themselves to one-sex genealogies, which simplified the problem quite a bit, not having to solve the complexity of the union formation and dissolution processes that a general ego-based model needs to simulate.

Ego-based models start directly with individuals and construct their complete genealogy without any previous information, like the observational data used in population-based model. This explains why ego-based models usually create backward genealogies in order to obtain the full kinship network, as a substitute for having to run the simulation for 100 or 200 years, as is needed in a model like SOCSIM. The simulation of the backward or ascending genealogy of egos is in fact the main difference with population-based models, which only incorporate an algorithm for simulating forward or descending genealogies. The algorithms for the generation of descendant kin also differ in part between the two classes of models, mainly for the formation of unions, with a mate-matching mechanism for population-based models, substituted by two-ways tables or functions of age at union by sex for ego-based models. The fertility and mortality modelling of backward generation of genealogies can be in principle more similar for population- and ego-based models, but fertility processes tend 
to develop over long time periods, and due to that are generally not completely simulated in fully-closed models, for which the synchronization of lifecycle events of the pool of individuals force to use shorter time periods.

\subsection{Le Bras' BACKFOR algorithm for the simulation of ascending genealogies}

Le Bras created the methodology for the construction of ascending or backward genealogies in ego-based models. When he developed the backward algorithm, at first it seemed to him to be simpler that the one needed for simulating forward genealogies, which involves modelling nuptiality and fertility processes. But this turned to be overly optimistic as we are going to see.

\section{a) First version: looking for ego's mother and then for her partner}

Le Bras (1973) started with an analytical model similar to Lotka's one (1931) for relating egos with their mothers, determining the probability of a mother to be alive taking into account her age at birth of ego, then relating in the same way the mothers with their grand-mothers, etc. But, contrary to Lotka (and Goodman et al. (1974) who later built on Lotka's model), Le Bras immediately posed the problem of the relationship of the children with their fathers, the fathers with the grand-fathers, etc. in order to obtain a complete genealogy. His solution was to introduce an asymmetry between ego's mother and father. He proposed to start relating egos with their mother and then to relate the mother with the father, instead of building a direct relationship between egos and their fathers. This is justified by the fact that the age at union of the mother and the father are not independent, and hence the distribution of their age at childbearing will be highly correlated. Le Bras then obtained an analytical formula for computing probabilities of having both ego's parents alive, which was already quite complex, but then totally unpractical for computing probabilities of having her or his two parents and four grandparents alive, not even mentioning the possibility of having the grandgrandparents entering the picture, so he decided to use microsimulation instead to compute these probabilities.

The algorithm starts with the random selection of a mother based on her age at birth of ego. In a general way the age distribution of mothers of a group of egos born in year $t$ is: 


$$
B(t)=\int_{\alpha}^{\beta} W(t-x) l_{t-x}(x) m_{t-x}(x) d x
$$

Where $B(t)$ is the total number of egos born in year $t, W(t-x)$ are the births of women in the population $x$ years earlier, $l_{t-x}(x)$ is the probability of surviving at age $x$ for women born in year $t-x$ and $m_{t-x}(x)$ is their risk of having a child at age $x$ in year $t$, for ages at childbearing varying between $\alpha$ and $\beta$.

Then the probability for one ego of having a mother of exact age $x$ at her/his birth will be of:

$$
\frac{W(t-x) l_{t-x}(x) m_{t-x}(x)}{B(t)}
$$

which simplifies nicely in a stable population to:

$$
l(x) m(x) e^{-r x}
$$

Where $l(x)$ and $m(x)$ are the constant probabilities for women of surviving to or having a child at age $x$ and $r$ is the intrinsic growth rate of the corresponding stable population, and $x \in[\alpha, \beta]$.

For the purpose of the subsequent discussion, it is important to understand the consequences of using formula [1] for the selection of a mother for ego: it is a mechanism based on births, so a woman will be selected if she had children (a woman without children by definition cannot be a mother of ego). Moreover, in a general way, women with high fertility will have a higher probability of being selected than those with low fertility. It is easy to explain this if we think in terms of births in any time interval: a woman with several children is more likely to be included than a woman with only one child.

Le Bras' backward algorithm in the microsimulation begin with the random selection of an age $x$ at childbearing for the mother, according to probability density function [2] (for a population with mortality and fertility variables in time) or [3] (for a stable population). The father is then selected in two more steps: first an age at union for the mother is randomly drawn (conditional on the mother being in union at least 9 months before age $x$ ), then the father is randomly attributed an age at union using a two-dimensional function of ages at union of men in relation to ages at union of women. The same backward algorithm can then 
be applied recursively to ego's mother and father to obtain the four grandparents, and then can be again applied to each grandparent to obtain the eight great-grandparents of ego.

\section{b. Second version: the BACKFOR algorithm}

Le Bras (1982) presents the version of his model for simulating complete genealogies, integrating a forward algorithm (based on a nuptiality and fertility models very similar to the ones used in KINFERT, which, as discussed before, was derived from Le Bras' model) and a modified version of the backward algorithm described previously. This paper, which is hardly cited as it was published in French in the proceedings of a congress, is nonetheless by far the best study on the analysis of kinship structures, comparing historic and contemporary populations. The paper also included an appendix with a complete specification of the model which allows for an independent implementation to reproduce its results, something that is truly unique.

The new version of the backward algorithm was meant to correct a flaw in the previous version, as stated by Le Bras: the selection of the father was slightly biased as no allowance was made of the possibility that he dies before the month of the ego's fecundation. The introduction of the BACKFOR algorithm not only provided a solution to this bias, but, above all, merges together the logic of the backward and forward microsimulations of kinship.

This new algorithm starts with the same steps than the previous version of the backward one: first we randomly select an age at birth for the mother of the ego, then her age at union, compatible with the previous age at birth selected, and finally an age at union for the possible father, based on a two-ways table of ages at union of men as a function of ages at union of women.

The BACKFOR algorithm then proceeds with a forward simulation, using the fertility model to simulate the descendance of the couple, which allows the program to check whether they can be the parents of ego. This will be the case only if the woman has a living birth at the age at childbearing that was selected in the first step. This will not happen if the man dies or a separation occurs beforehand, and no compatible second union is formed later, nor if the woman had no children or had them at other ages than the selected age at childbearing. The woman is rejected if its descendance is not compatible (no birth at the age at childbearing 
selected), then the algorithm is executed again, starting with the selection of a new age at union for the man, until the model can simulate a compatible descendance for the current woman.

Figure 4 summarises how the BACKFOR algorithm proceeds to reconstruct the ascending genealogy of egos. It starts with a backward (BACK) simulation and then continues with a forward (FOR) one until it reaches a solution, which justifies its name. Specifically, it starts with one ego (individual 1 of the genealogy), then proceeds with his or her mother (individual 2) and then the mother's partner who could be the possible father we are looking for (individual 3). The descendance of the couple is then simulated and selected if the sib group includes a child born in year $t$. Therefore BACKFOR creates the group of siblings of ego in the same step. In turn, the same algorithm can then be applied to ego's mother and father in order to find compatibles grandparents for ego, which also allow to obtain the aunts and uncles of ego in the same step (for example, individual 7). The forward part of the algorithm can then be applied on them to construct the group of first cousins (not represented in this genealogy). The following step is to simulate the descendance of ego, which start with the selection of a partner (individual 9), the simulation of the descendance of the couple for obtaining the children (individuals 10, 11 and 12), whereby we also obtain the children-in-law (here individual 13) and the grandchildren (represented by individual 14) applying the forward algorithm to each child. There is no a priori limit on the depth of this genealogy, which can be extended at will in a recursive way, upward (for example with the group of greatgrandparents and of the great-aunts and great-uncles), forward or laterally.

Results of Le Bras' BACKFOR algorithm, as implemented in our model for comparison purposes, are presented in later sections. 
Figure 4. Le Bras' BACKFOR algorithm for the construction of ascending genealogies
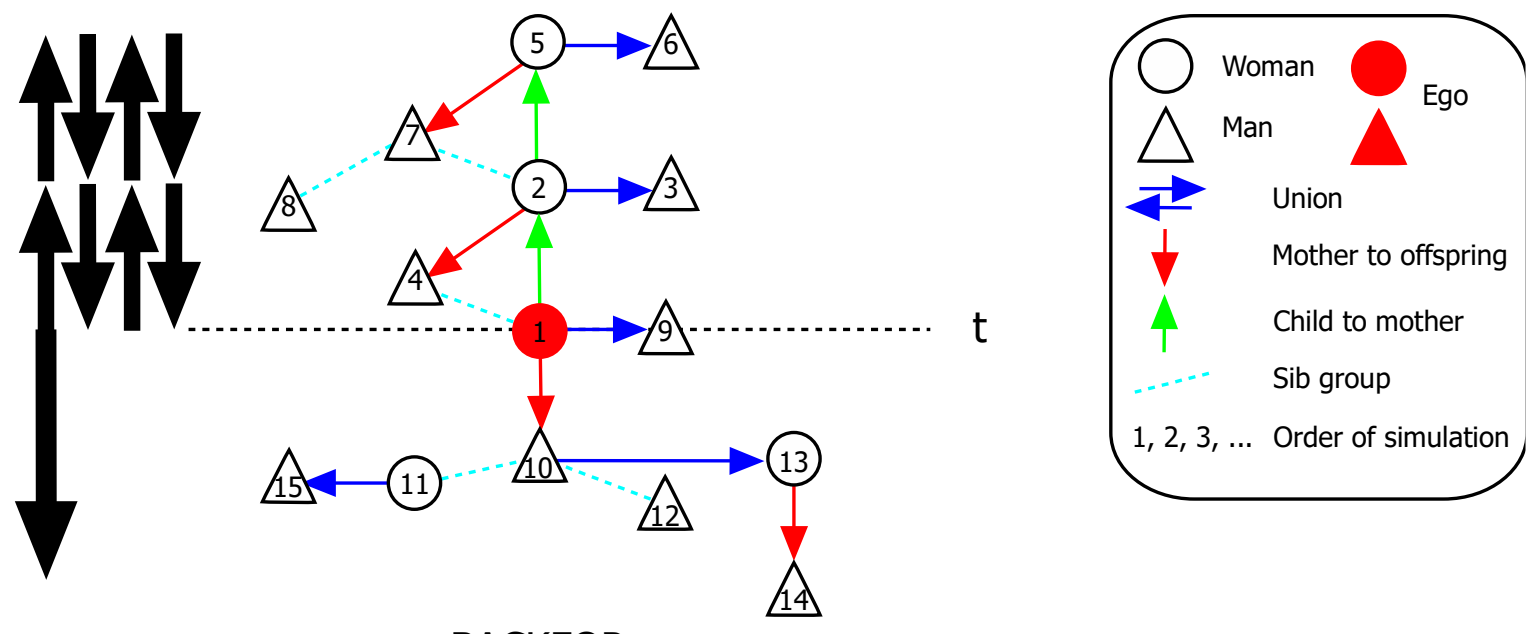

BACKFOR

Note: the BACKFOR algorithm, as described in Le Bras (1982), is pictured by the upward and downward arrows on the left: first we go upward from an ego to a putative mother, then downward with the reconstruction of the maternity history of this woman. We repeat these two steps until we obtain a mother with a birth at the correct age.

\subsection{Cambridge's CAMSIM}

SOCSIM was developed in part to address demographic issues related to the emergence of societies based on nuclear families (households limited to a couple and their children). Laslett (1965) established, against the prevailing sociological views at the time, that extended-type households were rare in England by the 16th century, and that the spread of the nuclear type was not the product of the demographic and economic changes associated with industrialisation in the $19^{\text {th }}$ century. Microsimulation of kinship was then used to respond to criticisms that showed, among other things, the commonness in the past of other household forms, such as the stem-family (households with kin of three generations: Berkner, 1972).

The first results of SOCSIM demonstrated that the prevalence of the nuclear type was not due to the high mortality before the $18^{\text {th }}$ century, which would have limited the co-residence of several generations.

The SOCSIM model was developed at the University of California, Berkeley, with the aim of adapting it to the computer systems at the University of Cambridge in the UK. But software incompatibilities and hardware limitations made it difficult to use SOCSIM there, as it requires the use of population lists that occupy a large amount of RAM memory. This led to the 
development of an alternative model, 'Cambridge Simulation' or CAMSIM (Zhao, 2006), that is more efficient in terms of computer resources. It is the best-known ego-based microsimulation model of kinship, although it is a direct descendant of Le Bras' BACKFOR model.

CAMSIM incorporates a fertility module that takes into account individual heterogeneity in fertility, and also the progression of infertility with age, but the fertility module is less detailed than that of Le Bras or the fertility simulation module of KINFERT. For example, it only takes into account the duration of intervals between births, and therefore not of the age pattern of fecundability. The algorithm for generating ascending genealogies also is based on questionable choices. Thus, it has had two different versions, but their descriptions are not very detailed, which forced us to make some guesses in order to carry out an independent implementation for checking its results, and be able to compare with those of Le Bras' model or with KINFERT's ones.

\section{a) First version of CAMSIM algorithm for generating ascending genealogies}

Smith (1987) presented the first version of CAMSIM (henceforth referred to as CAMSIM_87), and in particular its implementation of BACKFOR, which is very rudimentary compared to Le Bras' model. The algorithm for finding a mother for ego is based simply on the repeated creation of a woman's union and reproductive history until she has at least one child. Ego is then selected by a random draw of a birth order. Smith states that the results are biased, because there is no mechanism for determining the year of birth of ego's mother, and thus no account can be taken of the past evolution of births, which is the basis of a correct random selection of ego's mother as established by Le Bras with formulas [2] or [3].

We will see later that this version of CAMSIM has in fact a much more serious bias, since the probability of selecting a mother depends only on whether or not she has a child, and not on her number of children, which leads to the selection of mothers with a lower fertility level than expected. 


\section{b) Second version of CAMSIM's BACKFOR algorithm (CAMSIM_93)}

Laslett (1988) and Smith \& Oeppen (1993) described a more elaborate later version of the algorithm for creating backward genealogies, which is much closer to Le Bras' model. CAMSIM_93 proceeds in two steps:

a) Random drawing of a fertility level for ego's mother according to the probability distribution of the associated stable population. A birth order for ego is then randomly selected (based on a uniform distribution).

b) Random drawing of an age at childbearing according to the age distribution of the corresponding stable population for women with the fertility level selected in the previous step.

CAMSIM_93 then applies its downward algorithm for creating a woman's reproductive history, which includes creating at least one partner who can be the father of ego. If this woman has the desired number of children, then we obtain the age at birth of the child from the order selected in the previous step, and if this age is identical to the required age at childbearing, then this woman is selected as the mother of ego. If she does not have the number of children sought, or if the child of the order selected was not born at the correct age at childbearing for the mother, then the algorithm is run again until a woman is found with a compatible reproductive history.

It can be seen that the algorithm now includes a mechanism to determine the year of birth of the mother. On the other hand, it presents an additional level of complexity compared to Le Bras' model which is not deemed necessary: no justification whatsoever is presented for picking a number of children before selecting an age at childbearing of the mother.

\section{c) Characteristics and limitations of the BACKFOR algorithm}

First, we can observe that the reconstruction of ascending genealogies is not only algorithmically different from descending genealogies, but a different probabilistic background is also used: as can be seen from the probability density functions (PDFs) in formula [2] or [3], the probability of having a mother of age $x$ is function of population characteristics, like the past number of births. This is only partly the case for descending genealogies, which use PDFs based on individual characteristics for the risks of having 
children, for the risks of separation of the couple, for the mortality risks, etc. Notwithstanding, it is important to note that the probabilities associated with union formation, and for example the construction in Le Bras' model of the two-ways table of ages at union of men, the function of ages at unions of women use also population characteristics including the past number of births. So even if ego-based models do not include directly a mate-matching algorithm, as closed population-based models do, they can take into account the population age structure to generate union formation PDFs, as we will also see later when we describe KINFERT, our version of an ego-based microsimulation model of kinship that was derived from Le Bras' model.3.38 Second, we can note that the BACKFOR algorithm has some benefits, like its simplicity and the small amount of memory needed to store the information on partners and children, but it is inefficient as it requires a large amount of computation time and also discards a lot of useful data on couples' fertility due to its rejection mechanism. The degree of inefficiency depends on the fertility level of the population simulated, and it is higher when this level is low, as the program spend more time looking for a compatible couple. In order to measure this inefficiency, and for comparison's sake, we implemented in our model the various versions of the BACKFOR algorithm described previously, different from the one we use in our model and that we describe later. Table 2 reports the results of 4 runs of Le Bras' BACKFOR algorithm as well as of the second version of CAMSIM, when reconstructing the genealogies of 10000 egos and as per the implementation of these algorithms in KINFERT. For example, with a fertility level of 2.11 children per women, Le Bras' version of BACKFOR needs to discard on average 142 women with their reconstructed descendance before obtaining one with the correct age at childbearing for ego. The inefficiency is lower for a high fertility level (discarding on average 9 reconstructions of fertility histories for a TFR of 3.64) but very high for a TFR of 1.29 children per woman (1878 histories discarded on average when looking for parents, and for each genealogy, when including the parents as well as the grandparents of ego, we need to multiply this number of rejections by a factor of 3 ). We observe also that the CAMSIM version of the BACKFOR algorithm is much more wasteful both in computing time and simulation results as it discards between two and ten times more BACKFOR simulations than Le Bras' version, due to the extra step added (previous selection of a number of children). 
Table 2. Mean number of BACKFOR tries in function of the fertility level used for the simulation of backward genealogies in Le Bras' and CAMSIM models

\begin{tabular}{|lcc|}
\hline TFR & Le Bras & CAMSIM (1993) \\
\hline $\mathbf{1 . 2 9}$ & 1879 & 4141 \\
\hline $\mathbf{2 . 1 1}$ & 143 & 642 \\
\hline $\mathbf{2 . 7 4}$ & 45 & 300 \\
\hline $\mathbf{3 . 6 4}$ & 10 & 115 \\
\hline
\end{tabular}

Note. TFR: level of the Total Fertility Rate entered as input for the microsimulation of kinship. Le Bras / CAMSIM: mean number of BACKFOR tries necessary to select the parents of individuals (for egos and for each one of their parents) in a stable population with our implementation of Le Bras' and CAMSIM' version (as described respectively in Le Bras, 1982 and Smith \& Oeppen, 1993). The mean number of tries reported is an underestimate of the true number, as we modify the age at childbearing to find a compatible couple in a reasonable time. For example, if the selected age at childbearing is low (or high) and we do not obtain a couple with a birth at the correct age after 10000 unsuccessful tries, we increase (or decrease) it by one year until the BACKFOR algorithm finds an adequate couple.

But the final reason why we chose not to use the BACKFOR algorithm in our model is that it unfortunately gives biased results, as it typically leads to select couples of parents with a higher fertility level than the correct value, as we show in a later section of this paper.

\subsection{Ruggles' MOMSIM model for generating household structures}

Ruggles (1987) sought to test the results obtained with SOCSIM regarding the demographic determinants of household composition and to confirm or invalidate Laslett's theory about the prevalence of the nuclear household in England at least as early as the 16th century. Ruggles' main criticisms concerned SOCSIM's assumptions about co-residence rules between family generations. In order to study their role in justifying or not Laslett's observations, he did not hesitate to construct a microsimulation model of kinship from scratch, which he named MOMSIM.

MOMSIM is not as well known as SOCSIM or CAMSIM, mainly because Ruggles did not distribute it, and abandoned its development a few years later, but it is nevertheless 
interesting, because it is different from all the models developed before, especially as regards the construction of ascending genealogies.

Figure 5 presents a schematic view of the model. Ascending genealogies covering at most three generations are constructed by downward simulations of the lifecycle of groups of women, represented here by individual 1 . She is first assigned a spouse (individual 2) and then children (3, 4 and 5). The model follows with the reconstruction of the downward kinship of the second generation and children 3,4 and 5 form a union respectively with 6,9 and 12, and they all have at least a child (respectively 7 and 8, 9 and 13) who are members of the third kin generation simulated. Then the model randomly assigns attributes to the grand-children of individual 1 , such as their age at death and whether they will have a partner later in life (for example individual 11 will be the spouse of 10), but without reconstructing further the descending genealogy which does not include members of the fourth generation (that would be great-grandchildren of 1 ).

\section{Figure 5. MOMSIM algorithm for the generation of genealogies}
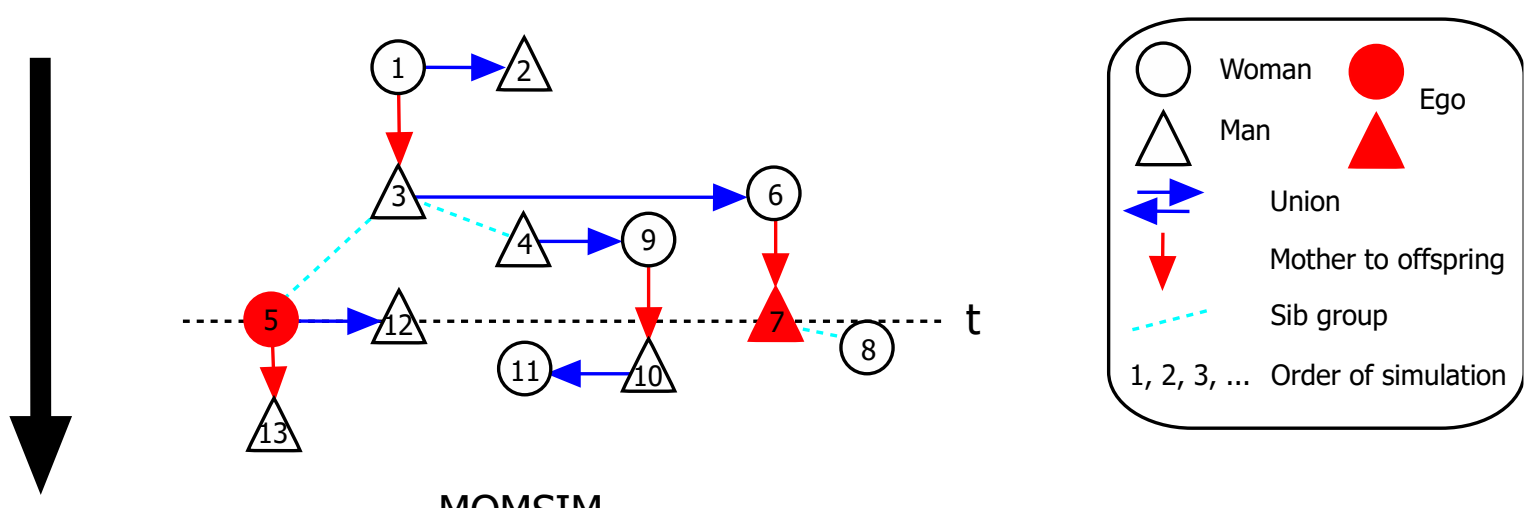

\section{MOMSIM}

The focus of Ruggles is on members of the second generation who during their life will form households that may possibly include their parents 1 and 2 and their children (a stem-family) and also possibly some of their siblings (an extended household). But if we focus on kin born in the same year (individuals 5 and 7 that we take as egos), we observe that 5 is an aunt for 7 and both have individual 1 in their respective ascending genealogy, as mother for 5 and grandmother for 7 . This circumstance is rare nowadays, in low-fertility societies, but was quite common in the past. The problem is that it leads to an overestimation of the expected numbers of siblings, as the genealogies with this kind of age-crossing of aunts-uncles with their nieces-nephews are more common in families with a high fertility level of the woman 1 
who is the root of the (inversed) tree. Therefore, the construction of ascending genealogies cannot be done following in a systematic way a downward genealogy backward if we want to obtain the correct probabilities or expected number of kin of a certain type, alive or not.

\subsubsection{Implementation of the kinship generating module of KINFERT}

\subsection{Generation of backward genealogies}

The construction of ascending genealogies in KINFERT is based entirely and literally on formula [1]: ego's mother is a woman who had a child in the year of her/his birth. Thus, if we want to obtain a mother whose characteristics respect the probability distribution indicated by this formula, we can complement the BACKFOR algorithm with a version of the MOMSIM procedure, but limiting it to a two-generations descending genealogy. The KINFERT algorithm for assigning the mother of an ego born in year $t$ then proceeds as follows:

a) Construction of the descending genealogies of women born between $t-\beta$ and $t-\alpha$. The number of these women will be proportional to the time series of past births, which may correspond to the sequence of births in a stable population, or to its actual evolution in the population under study. This corresponds in the following figure to the downward arrow in the direction of the passage of time, at the extreme left: the reconstruction of descending genealogies is limited to two consecutive generations, those of the mothers and egos' one.

b) Selection of all births of previous genealogies which occurred in year $t$.

c) Assignment of a mother for an ego born in year $t$ by a random draw in the birth table built in the previous step: uniform random selection of a number from 1 to the size of this table (this assignment corresponds to the green upward arrow in the figure, linking ego and her/his mother).

This algorithm is an adaptation of the BACKFOR mechanism, in which none of the simulated reproductive histories are discarded, which allows considerably simplifying ego's mother selection procedure. With KINFERT, the mother and her age at ego's birth are selected at the same time, by a random draw in the birth table for year $t$. In Le Bras model or for CAMSIM, there is an additional step required in order to simulate the current woman's descendance, in the search for a compatible mother, which is the random assignment of an age at first union lower than the age at maternity selected at the previous step according to PDFs [2] or [3]. As 
we shall see in the following, this additional step is the source of an important bias and therefore a drawback for the use of BACKFOR, at least in its version corresponding to Le Bras or CAMSIM model.

In all fairness, nowadays this adaptation of BACKFOR is possible because computer systems dispose of huge amounts of RAM memory which allow ego's possible mother's union and reproductive histories to be stored, a luxury that was not available to Le Bras and Smith in the 1980s.

Figure 6. KINFERT algorithm for the construction of complete genealogies of egos
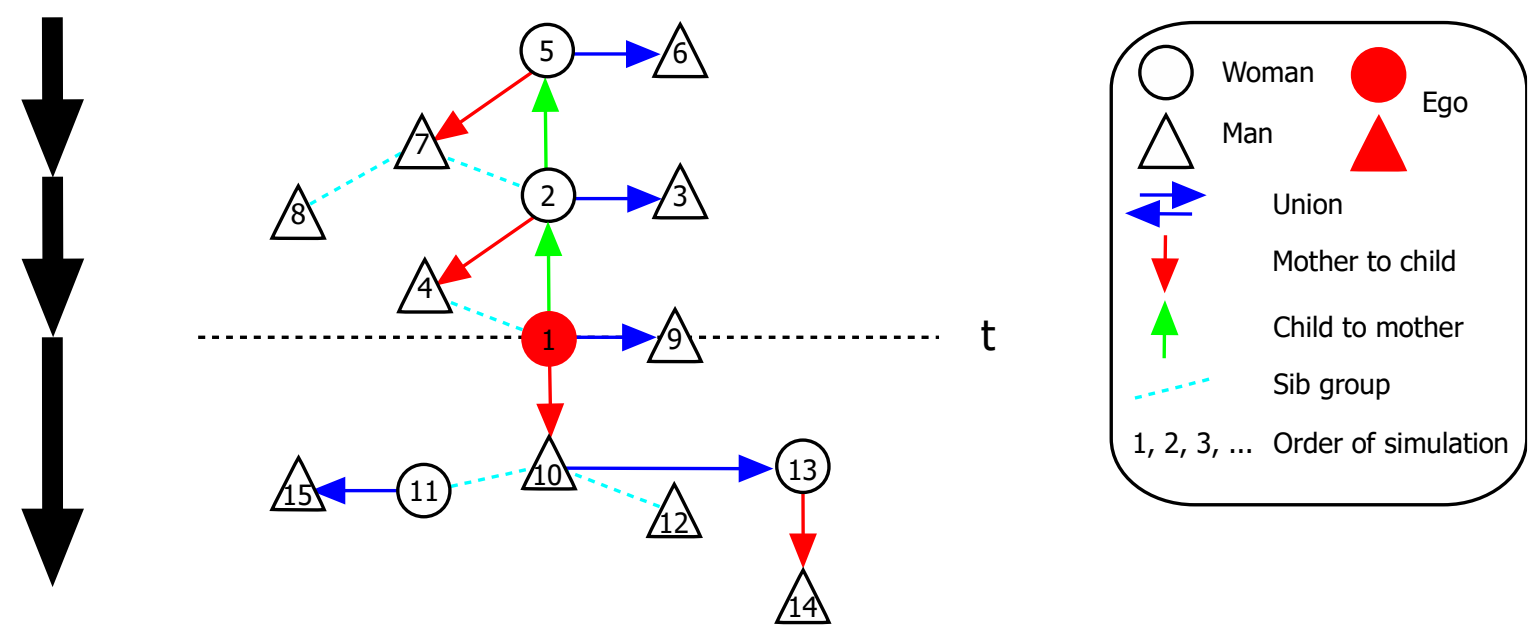

KINFERT

\subsection{Looking for ego's partners}

Another advantage of keeping information on the family cycle of women, as described in the previous point, is that the corresponding union histories are also available, which facilitates the search for a partner for ego, and also provides data on the life segment prior to the current union. KINFERT is an open model, in the sense that at the time of union formation, a partner is created without restriction. This is not the case in a closed model, where a union will only occur if the pool of compatible candidates allows it, and if this is not the case, the model will put the unhappy candidate in a queue. However, KINFERT also takes into account the age composition of the population through the following algorithm:

a) The simulation of the life of a male ego, born in year $t$, begins with a random draw of an age at death, then of a probability of forming or not at least one union and of the 
corresponding age at first union in case ego does not remain single. If this second age is lower than the first, then a female partner is sought among all women whose union and reproductive history has been simulated for the preceding algorithm for assigning a mother to each ego. This means, however, that this simulation must be extended into the future in order to have the possible unions up to $t+M$, where $M$ is the maximum age at union considered.

b) The simulated union histories for these women include the ages of all her partners at the time of the respective unions, allowing the creation of a two-entry table with the year of birth and corresponding age at union of these male partners, with each cell containing a vector of references to women's histories.

c) The year of birth of ego and his age at first union are then used to select a woman from the previous two-entry table, by a uniform draw on the number of women in the corresponding cell (if the vector in the cell is empty, the table is explored in order to search for a union at the same age for the man and for another generation).

d) The life history of the selected woman that predates the current union is retained, allowing for the possible inclusion of other previous unions and children, and the model creates a new reproductive history for the current union.

KINFERT therefore creates union and reproductive histories for successive cohorts of women of a size proportional to the births that would be observed in a stable or real population, and these histories make it possible to find both mothers for all egos, and also partners for the male egos. In the current version of the model, there is no symmetrical simulation of union and reproductive histories for male cohorts, which leads to ignoring the previous unions and fertility history of male partners of female egos, before their current union, and also to not take into account past birth trends. This restriction will be lifted in a future version of the model, as the only limiting factor for not doing it is the available amount of free RAM memory for storing the corresponding male life history tables at runtime. 


\subsubsection{A criterion for judging the correctness of the results of a backward microsimulation of} kinship

It is important to be able to check the correctness of the results of a microsimulation, but in the case of a kinship reconstruction, the main problem is that there is hardly any observational data to perform an external type of test of the complete genealogies, in particular the ascending part (parents and grandparents) or the lateral part (first cousins). On the other hand, it is easier to conduct such a test if one limits oneself to descending relatives (children and grandchildren), as was done for example by Wachter, Blackwell, \& Hammel (1997) when they compared past results of SOCSIM with data from a survey with specific questions on close family members (siblings, children and grandchildren). However, no test has been conducted on the goodness of fit of the results of microsimulation models of kinship concerning their ascending part. We propose here such a test, of an internal type, based on the functional relationship that should be observed between the fecundity of a generation of egos and the generation of their mothers.

One of the main results of an individual microsimulation of kinship are the probabilities that egos will have relatives of some kind alive (or the expected numbers if ego can have more than one relative of a specific kind). These are probabilities or expected numbers "from ego's point of view". This observation makes it possible to construct a test of the correctness of the results of a backward microsimulation, in the case where the demographic regime is stable (which means, among other things, that the mortality and fertility of female egos will be identical to that of their mothers' generations). Indeed, the genealogies obtained allow the direct calculation of the fertility levels used in the simulation. We can thus calculate the level of fertility of female egos (using only genealogies for which ego survives at least up to age 45):

$$
m=\sum_{i=0}^{K} p_{i} i
$$

Where $p_{i}$ is the proportion of women who have $i$ children at the end of their fertile lives (and therefore $\left.\sum_{i=0}^{K} p_{i}=1\right)$ and $K$ is the maximum number of children, $m$ is the average number of children of female egos, equal to the Total Fertility Rate (TFR), which is normally calculated 
from age-specific fertility rates. By comparison, the fertility of potential mothers of egos (male and female) will be equal to (again retaining surviving women up to the age of $45^{4}$ ):

$$
m_{1}^{\text {mothers }}=\sum_{i=1}^{K} q_{i} i
$$

$m_{1}^{\text {mothers }}$ is the average number of children of egos' mother, i.e. women with at least one child, a number always higher than $m$ because it excludes women who had no children, and therefore cannot be included in the genealogies of egos. The relationship between these two measures of fertility is as follows:

$$
m_{1}^{\text {mothers }}=\frac{m}{1-p_{0}}
$$

Where $p_{0}$ is the proportion of women without children. The relationship between the two types of proportions of women by number of children is identical, as:

$$
q_{i}=\frac{p_{i}}{1-p_{0}}
$$

Where $q_{i}$ is the proportion of mothers with $i$ children (and $\sum_{i=1}^{K} q_{i}=1$ ).

If we now try to calculate the fertility of egos' mother "from ego's point of view", i.e. by tracing the genealogies back starting from each ego, we will obtain an average number of children for mothers of egos, higher than $m_{1}^{\text {mothers }}$, which in contrast is a measure of fertility "from egos' mother point of view". The fertility level of egos' mothers, from egos' point of view is (again limiting to genealogies in which ego's mother is alive at age 45):

$$
m_{1}^{e g o s}=\frac{\sum_{i=1}^{K} q_{i} i^{2}}{m_{1}^{\text {mothers }}}
$$

It can be seen that in the numerator the number of children is squared, as we are now calculating "from the point of view of children". It is easy to understand why it is so from an example. If we consider two mothers who had respectively 1 and 3 children, the level of fertility "from mothers' point of view" $m_{1}^{\text {mothers }}$ will be 2 children. But the result "from children's point of view" will be different, since the 3 children of the second woman will each 
answer "my mother have 3 children" and the value of $m_{1}^{e g o s}$ will be $\frac{0.5 * 1^{2}+0.5 * 3^{2}}{0.5 * 1+0.5 * 3}=\frac{1^{2}+3^{2}}{1+3}$ or 2.5 children. Formula [4] can be simplified:

$$
m_{1}^{e g o s}=\frac{\left(\sum_{i=1}^{K} q_{i} i^{2}\right) /\left(1-p_{0}\right)}{\left(\sum_{i=1}^{K} q_{i} i\right) /\left(1-p_{0}\right)}=\frac{\sum_{i=1}^{K} p_{i} i^{2}}{m}
$$

Moreover, if we observe that the variance of the number of children is:

$$
\sigma_{m}^{2}=\sum_{i=0}^{K} p_{i}(i-m)^{2}=\sum_{i=0}^{K} p_{i} i^{2}-m^{2}
$$

And as $\sum_{i=0}^{K} p_{i} i^{2}=\sum_{i=1}^{K} p_{i} i^{2}$, we finally obtain the formula that allows us to judge the correctness of a backward reconstruction:

$$
m_{1}^{e g o s}=m+\frac{\sigma_{m}^{2}}{m}
$$

This formula states that the fertility level of egos' mother, calculated from backward simulated genealogies, which are by definition constructed "from the point of view of ego", will be equal to the fertility level of female egos plus the variance of their number of children divided by their average.

It is interesting to note that formula [5], which is a measure of fertility, is equal to the classical formula for calculating the average size of the sibling group (Preston, 1976), which using our notation can be written as :

$$
S G=m_{1}^{\text {mothers }}+\frac{\sigma_{m_{1}^{\text {mothers }}}^{2}}{m_{1}^{\text {mother }}}=m+\frac{\sigma_{m}^{2}}{m}
$$

It is also similar to the expected number of siblings of ego (Goodman, Keyfitz, \& Pullum, 1975):

$$
S=m+\frac{\sigma_{m}^{2}}{m}-1
$$

Finally, it should be observed that formula [5] will be exact only if the intrinsic growth rate of the stable population is null, because we are comparing the fertility of egos born in one year to the fertility of their mothers born across a range of around 20 years. If the growth rate is positive, then women of older birth cohorts will be proportionally underrepresented compared to younger women and the backward algorithm will overrepresent lower age at childbearing, which are more likely to correspond to women with a lower number of births. In order to take this effect into account, a correction factor will be applied to observed values 
of $m_{1}^{e g o s}$ when using formula [5] to check the correctness of different algorithms of backward reconstruction of genealogies:

$$
\widehat{m_{1}^{e g o s}}=m_{1}^{\text {egos }} e^{1.2 r}
$$

where $\widehat{m_{1}^{e g o s}}$ is the value we will compare to $m+\frac{\sigma_{m}^{2}}{m}$ and $r$ is the intrinsic growth rate of the stable population. The value of 1.2 is arbitrary and based on the comparison of results of different runs of KINFERT.

\subsubsection{Comparing the results of the simulation of ancestors in different microsimulation models of kinship}

We ran the model with 5 different fertility levels, under stable population conditions with a very low mortality level (life expectancy at birth of 100 years for women and 98 years for men), with fertility levels ranging from 1.3 up to 4.7 children per woman. 10,000 ascending genealogies that include parents and grandparents were generated using the algorithms of Le Bras model, the two versions of CAMSIM one and of KINFERT. The results are compared in Figure 7, which shows the relationship between the fertility level entered as input and the fertility of ego's mothers calculated from the genealogies generated by each backward algorithm, after applying the correction factor [6]. Observed values of $\widehat{m_{1}^{\text {egos }}}$ are then compared to the theoretical level that should be recovered from the genealogies.

It can be seen that the model whose results are furthest from the correct values is the first version of CAMSIM (Smith, 1987). Its algorithm is based on the direct selection of a mother and not of one of her births, which is the correct procedure in probability, as shown by formula [1]. In fact, the fertility level of egos' mother in CAMSIM_87 corresponds exactly to the value of $m_{1}^{\text {mothers }}$, i.e., the fertility level from mothers' point of view, much lower than the level from egos' point of view.

The results of models using the Le Bras' or CAMSIM_93 BACKFOR algorithm are closer but tend to overshoot the correct value. This upward bias is a consequence of a critical flaw in this algorithm: the second step consists of choosing an age at first union for the woman that is lower than the age at maternity selected before. This random selection is therefore conditioned by a maximum level, but not by the fact of having had a child at this age, which 
leads to the selection of an age at union that is generally too low, which explains in turn why the women selected as mothers will have a higher fertility level than expected. Unfortunately, there is no solution to this, and thus the original BACKFOR algorithm is fatally doomed as a solution for generating ascending genealogies.

Finally, we observe that our model always recovers the correct value for the fertility of mothers, when generating backward genealogies.

Figure 7. Fertility levels of egos' mother in different models (computed from genealogies), compared to the theoretical level

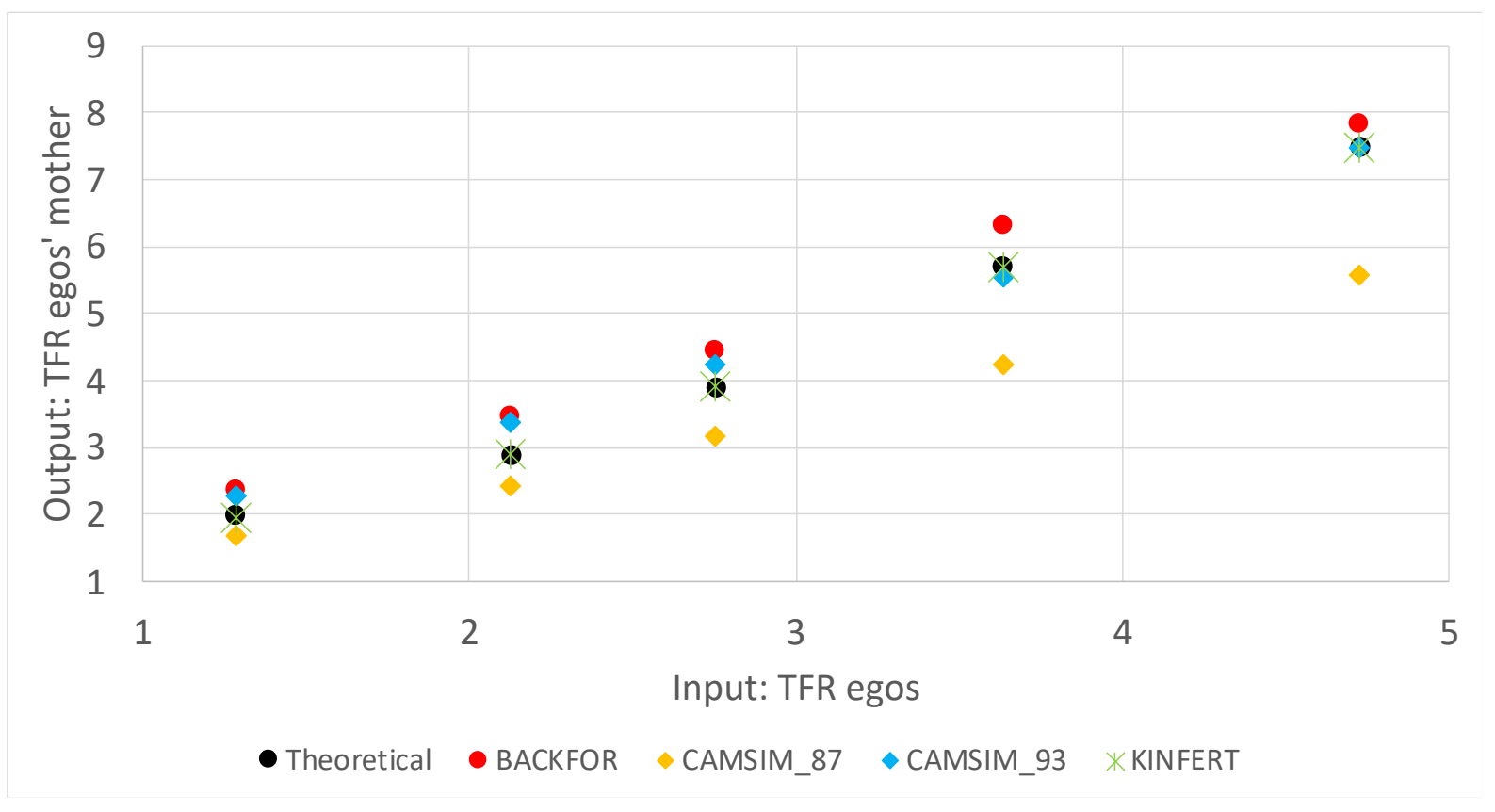

Note: results of 5 runs of KINFERT compared to 5 runs of our implementation of Le Bras' BACKFOR and of the two versions of CAMSIM model, with the same set of 5 different constant levels of TFR as input, and a constant and very low mortality level (stable population settings). Theoretical corresponds to the level of TFR for egos' mother, from ego's point of view, computed from formula [5], BACKFOR, CAMSIM_87, CAMSIM_93 and KINFERT are the corresponding levels obtained from the genealogies generated by these models, after applying correction factor [6]. 


\section{The Agent-Based module of the model}

More specifically, our model follows a group of approximately 10,000 egos from different birth cohorts, whereby the demand for care is studied from the age of 50 until death. The kinship network of egos is reconstructed, limiting it to spouse, children, children-in-law, and grandchildren. This is obtained from the KINFERT kinship microsimulation model which recreates the whole diversity of family situations. At this stage, the basic lifecycle of all individuals is reconstructed, giving them an age at union formation (if this is the case), an age at the birth of each child (if this is the case), and finally age at death. The model generates kin networks in an 'open' way, in the sense that there is no constraint on the stochastic 'creation' of kin other than the general fertility, mortality, and union formation risks.

For this article, we compare seven different generations of egos, born at intervals of 10 years, between 1908 and 1968, with very different demographic characteristics (see Table 3). In our model the members of the close kinship network of egos of each generation are individuals who belong to other birth cohorts and therefore have nuptiality and fertility behaviour, as well as mortality risks, that differ from those of egos. In other words, our kinship model does not assume that the population is 'stable, but, on the contrary, that each birth cohort has its own demographic characteristics. This is important, especially with more recent cohorts for which the fertility levels of children are less than half those of their parents. The ABM then estimates the demand for care of these egos that could be satisfied by the close kin network (spouse and children). When this is not possible, either totally or partially, the ABM calculates the proportion of the care demand that cannot be taken on by informal family resources and that should, therefore, be externalized, presumably to the formal sphere. The model allows estimating and projecting the demand for care of the elderly in Spain and the distribution of care of a formal (professional) or informal type (by family members) according to the demographic characteristics of each birth cohort, as well as comparing the different Spanish generations born during the twentieth century.

With the aim of only identifying the impact of demographic change, we hold constant all the other factors considered in our simulation. For example, the current $A B M$ is based on the health conditions and employment status observed in 2008, which are invariable for all generations. Specifically, the model constrains the age-specific health conditions of each 
cohort to the dependency levels derived from the 2008 Spanish Survey on Disabilities, Personal Autonomy and Situations of Dependency (EDAD). This is a reasonable assumption given the lack of evidence that morbidity is being compressed in Spain, as mentioned above. Introducing the possibility for age-specific cohort changes in dependency levels, as well as employment status, however, will be considered in a future version of the model.

Table 3. Demographic trends in Spanish cohorts born in 1908, 1928, 1948 and 1968

\begin{tabular}{lcccc} 
Cohort & $\mathbf{1 9 0 8}$ & $\mathbf{1 9 2 8}$ & $\mathbf{1 9 4 8}$ & $\mathbf{1 9 6 8}$ \\
\hline $\mathrm{e}_{0}$ (life expectancy at birth, women) & 49 years & 55 years & 72 years & 85 years \\
e 65 (life expectancy at age 65, women) & 11 years & 12 years & 16 years & 23 years \\
Proportion of survivors at age 65, women & $40 \%$ & $49 \%$ & $78 \%$ & $95 \%$ \\
Definitive singlehood, women & $14 \%$ & $11 \%$ & $5 \%$ & $8 \%$ \\
Female widowhood at age 50 (\%) & $23 \%$ & $19 \%$ & $12 \%$ & $1 \%$ \\
Male widowhood at age 50 (\%) & $12 \%$ & $7 \%$ & $3 \%$ & $3 \%$ \\
Total fertility rate (TFR) & 2.7 & 2.5 & 2.3 & 1.5 \\
Childlessness (\% of women) & $25 \%$ & $18 \%$ & $13 \%$ & $21 \%$ \\
Age at first motherhood & 27 years & 27 years & 26 years & 30 years \\
Women who have at least 1 child (\%) & $75 \%$ & $82 \%$ & $87 \%$ & $79 \%$ \\
2 children (\%) & $65 \%$ & $70 \%$ & $76 \%$ & $57 \%$ \\
3 children (\%) & $44 \%$ & $41 \%$ & $35 \%$ & $13 \%$ \\
4 children $(\%)$ & $29 \%$ & $24 \%$ & $16 \%$ & $3 \%$ \\
5+ children (\%) & $20 \%$ & $14 \%$ & $7 \%$ & $1 \%$
\end{tabular}

Source: Mortality indicators and value of the mean age at first motherhood are the authors' calculations based on data from the National Institute of Statistics (www.ine.es). Fertility values, total and by birth order, are from Devolder (2018). The rest are estimates obtained from our kinship microsimulation model. See also Spijker et al (2020).

The egos of the seven cohorts born between 1908 and 1968, as well as their kin, are exposed in the $A B M$ to the risks of falling into a specific dependency level, as observed for Spain in 2008. Dependency is specified in terms of four levels: high, medium, low, or none. This distinction was made because the EDAD data showed clear differences in the (hours of weekly) care received according to dependency level. To be more specific, highly dependent individuals are those who report major difficulties in carrying out, without help or supervision, at least two Activities of Daily Living (ADL) related to everyday personal care. ${ }^{5}$ Adults with medium dependency report difficulty in carrying out one ADL or one or more Instrumental

\footnotetext{
${ }^{5} \mathrm{ADL}$ has the following items: changing or maintaining body position, moving around the houses, washing oneself or caring for body parts, toileting, dressing and undressing, eating, and drinking
} 
Activities of Daily Living (IADL) without help or supervision. ${ }^{6}$ People with low dependency have a disability but no ADL or IADL difficulties. Although we assume in our model that they do not receive care (as according to the 2008 EDAD data only $9 \%$ of low-dependent adults did receive it), we separated them from the disability-free population as they are less likely to provide care (see also Table 5). Level-specific dependency risks depend on the ego's age and sex, as well as educational attainment, given the sharp educational gradient in dependency. Hence, as Figure 8 shows, less-educated individuals are more likely to have a high degree of dependency (implying more hours of care required) than those with a higher educational level. Likewise, women are at much greater risk of falling into a state of dependency than men. For example, the proportion of dependent women in the 85-89 age group is around 15 points higher than that of men for all educational groups. For the ABM, we derived from the preceding proportions a set of age-sex-education specific transition probabilities between the initial situation of good health and the three dependent states of increasing severity, without considering the possibility of recovery. These probabilities are used both for egos and their relatives, with the aim of determining which part of our virtual populations requires care and to what degree.

The same logic is used to ascertain whether egos and their relatives work, this being based on activity tables that are a function of sex, age, educational level and degree of dependency. These tables are also obtained from the 2008 EDAD survey, and the probabilities of transition from one employment status to another are estimated from longitudinal data from the Spanish Labour Force Survey (EPA), using the second quarter waves of the years 2008 and 2009. Table 4 presents a summary of this information. As can be observed, labour force participation varies significantly by sex and educational level, as expected, while dependent people with a high educational level do not reduce their activity to the same degree as counterparts with a lower educational level. This justifies taking gender and education into account in our modelling.

We used estimates made by Noble et al. (2012) as a guide to determining the number of hours per week that dependent individuals require or can be offered to dependents by family

\footnotetext{
6 The questionnaire distinguished the following IADL items: taking medication, doing grocery shopping, preparing meals, and doing housework
} 
members, which is to say the informal care offer (Table 5), because estimates for the average number of hours of care based on the 2008 EDAD survey data, were very similar. The amount is a function of the age of kin and their employment situation as well as the state of their own health (level of disability or dependence).

The ABM determines, at each moment of ego's life, the situation of disability and dependence, as well as the number of hours of care that could potentially be received from the closest relatives who are still alive or, in other words, a partner and children. These can take care of ego as long as they have free hours, which can be limited by their work, state of health (but only if they have a low dependency status for, otherwise, they cannot provide care according to our model), and also by the needs for care of the rest of the family. For example, if ego has a disability but has a partner in good health who does not work, the latter will fully care for ego as long as their children are not in need of care as well, because of their level of dependency or due to their young age. On the other hand, ego's children will be able to devote care time to the extent that their own children do not require their time, again because of their age or a possible disability situation. In other words, the hours of care egos can obtain from their relatives are in competition with the needs of the other family members. This competition mechanism is implemented in the model through an algorithm of distribution of available hours of care based on simple rules, which are applied hierarchically to determine the number of hours of care that family members can offer to egos:

Figure 8. The proportion of the population in the highest dependency state (2+ADL) by sex, age and educational level (2008)

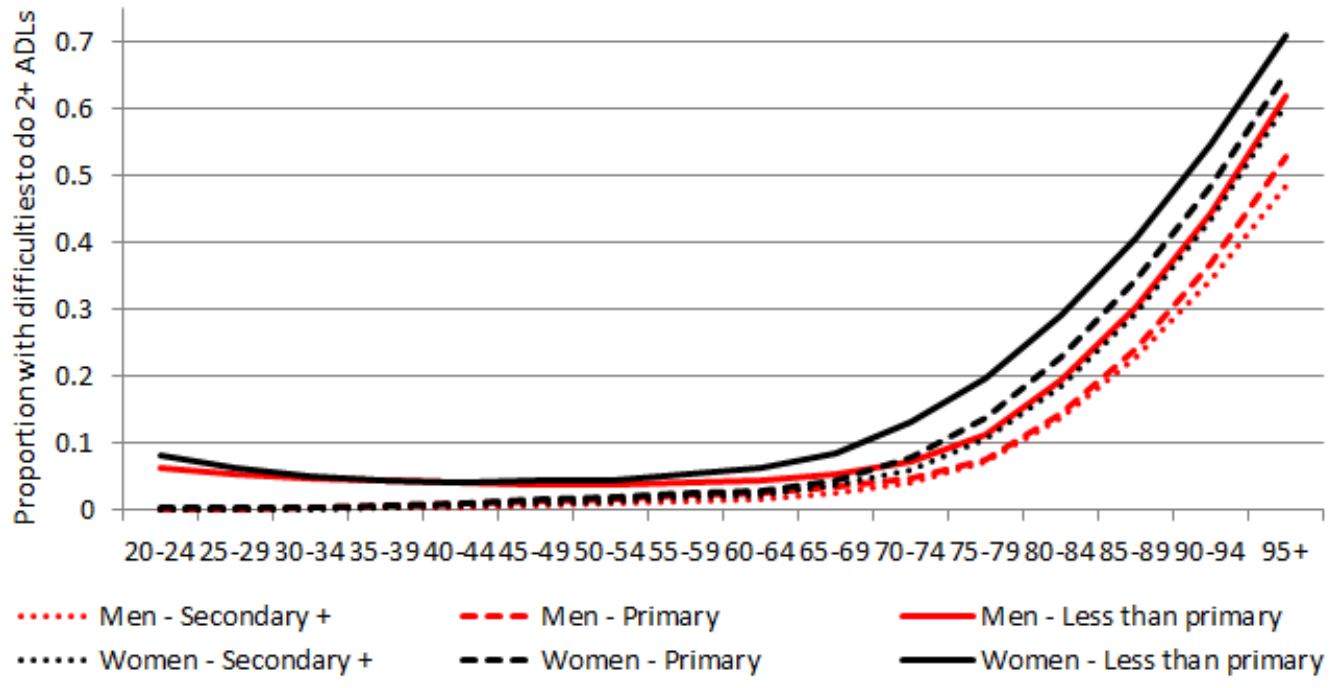

Source: Authors' calculations based on data from EDAD (2008). See also Spijker et al (2020). 
Table 4. Distribution of the population aged 16-69 by labour force participation according to sex, educational level, and level of dependency (2008)

\begin{tabular}{|c|c|c|c|c|c|c|}
\hline \multirow[t]{2}{*}{$\operatorname{Sex}$} & \multicolumn{3}{|c|}{ Men } & \multicolumn{3}{|c|}{ Women } \\
\hline & \multicolumn{3}{|c|}{ Educational level } & \multicolumn{3}{|c|}{ Educational level } \\
\hline $\begin{array}{l}\text { Dependency level/labour force } \\
\text { participation }\end{array}$ & $\underset{\%}{\operatorname{High}}$ & $\begin{array}{c}\text { Mediu } \\
\text { m } \\
\%\end{array}$ & $\begin{array}{c}\text { Low } \\
\%\end{array}$ & $\underset{\%}{\operatorname{High}}$ & $\begin{array}{c}\text { Mediu } \\
m \\
\%\end{array}$ & $\begin{array}{c}\text { Low } \\
\%\end{array}$ \\
\hline \multicolumn{7}{|l|}{ Disability with high dependency ${ }^{a}$} \\
\hline Inactive & 80 & 89 & 95 & 81 & 94 & 95 \\
\hline Part-time employed & 6 & 3 & 2 & 7 & 2 & 2 \\
\hline Full-time employed & 13 & 8 & 3 & 12 & 4 & 3 \\
\hline \multicolumn{7}{|l|}{$\begin{array}{l}\text { Disability with medium } \\
\text { dependency }^{b}\end{array}$} \\
\hline Inactive & 68 & 76 & 94 & 70 & 84 & 94 \\
\hline Part-time employed & 11 & 9 & 2 & 10 & 6 & 3 \\
\hline Full-time employed & 21 & 14 & 5 & 20 & 10 & 3 \\
\hline \multicolumn{7}{|l|}{ Disability with low dependency ${ }^{c}$} \\
\hline Inactive & 44 & 65 & 77 & 54 & 76 & 90 \\
\hline Part-time employed & 21 & 13 & 9 & 20 & 11 & 4 \\
\hline Full-time employed & 35 & 22 & 14 & 26 & 13 & 6 \\
\hline \multicolumn{7}{|l|}{ Without disability } \\
\hline Inactive & 23 & 29 & 46 & 38 & 60 & 77 \\
\hline Part-time employed & 3 & 3 & 2 & 13 & 13 & 9 \\
\hline Full-time employed & 74 & 69 & 52 & 49 & 28 & 14 \\
\hline
\end{tabular}

Source: Authors' calculations based on data from the 2008 EDAD survey. See also Spijker et al. 2020. Note: The three dependency levels are related with having a disability and not being able to carry out certain activities of daily living ( $A D L)$, e.g. dressing, and instrumental activities of daily living (IADL), e.g. cooking: ${ }^{a} 2+A D L{ }^{b} 1 A D L$ and/or any IADL 'Disability reported but no problems stated in carrying out ADL/IADL. Some of the totals do not add up to $100 \%$ due to rounding.

Table 5. Weekly hours of care demand (-ve values) and supply (+ve values), according to age, labour force participation (LFP), and dependency status used in the ABM model

\begin{tabular}{l|c|c|ccc} 
Age group & LFP & $\begin{array}{c}\text { Without } \\
\text { disability }\end{array}$ & \multicolumn{3}{|c}{ Dependency status } \\
\hline Less than 5 years & Inactive & -20 & Low & Medium & High \\
5 to 11 years & Inactive & -10 & -20 & -30 & -80 \\
$12-15$ years & Inactive & -5 & -16 & -30 & -80 \\
Adults & Inactive & 60 & 30 & -30 & -80 \\
& Part-time & 45 & 22.5 & -30 & -80 \\
& Full-time & 30 & 15 & -30 & -80
\end{tabular}

Source: Rough estimates based on Noble et al. (2012) and data from the 2008 EDAD survey. See also Spijker et al. 2020. Note: For definition of dependency statuses, see Table 2. 
1. Unmarried siblings, children or grandchildren of egos, help each other, before looking after their parents or receiving hours of care from them.

2. Parents preferably help their children, if they need care, before helping their partner or their own parents.

3. Ego's children will help their partners first, if they need it, before caring for their parents.

4. If ego and her/his partner are both in a situation of dependency, the hours of care their children can provide will be divided in proportion to the demand of each.

5. The care of an ego with a living partner will be carried out first and foremost by the partner, and ego's children will only contribute if the partner's offer of hours is insufficient to cover the demand.

Note also that, in the current version of the model, children-in-law care for their partner as well as for their own children, but not for their parents-in-law, and hence not for ego. The reason for this limitation is that the simulated kinship networks do not include the parents of children-in-law whose demand for care could also compete with ego's demand.

\section{References}

Andersson, G. (1997). The impact of children on divorce risks of Swedish women. European Journal of Population = Revue Européenne de Démographie, 13(2), 109-145. Retrieved from http://www.ncbi.nlm.nih.gov/pubmed/12293089

Bacon, B., \& Pennec, S. (2007). APPSIM - Modelling Family Formation and Dissolution. In NETSEM Working Paper. Retrieved from www.natsem.canberra.edu.au

Barrett, J. C. (1978). Effects of Various Factors on Selection for Family Planning Status and Natural Fecundability: a Simulation Study. Demography, 15(1), 87-98.

Berkner, L. K. (1972). The stem family and the developmental cycle of the peasant household : an eighteenth century Austrian example. American Historical Review, 77(2), 398-418.

Billari, F. C., \& Prskawetz, A. (2003). Agent-based computational demography: using simulation to improve our understanding of demographic behaviour.

Brüederl, J., \& Diekmann, A. (1995). The Log-Logistic Rate Model. Two Generalizations With an Application to Demographic Data. Sociological Methods \& Research, 24(2), 158-186. 
Calduch N, Spijker J, Zueras P, Giménez A, Griera O, Miguel Quesada F and Devolder D (2017) Application of the Overview, Design concepts and Details (ODD) Protocol to describe the DEMOCARE Agent Based Model. Papers de Demografia, 456.

Carrière, Y., Keefe, J., Légaré, J., Lin, X., \& Rowe, G. (2007). Population aging and immediate family composition: Implications for future home care services. Genus, 63(1-2), 11-31.

Coale, A., Demeny, P., \& Vaughan, B. (1983). Regional Model Life Tables and Stable Populations (Second Edi). New York: Academic Press.

Coale, A. J., \& McNeil, D. R. (1972). The distribution by Age of the Frequency of First Marriage in a Female Cohort. Journal of the American Statistical Association, 67(340), 743-749.

Devolder D (2002) Effects of the European late marriage pattern on kinship. A study using a microsimulation model. In Derosas R and Oris, M (eds), When dad died. Individuals and Families Coping with Family Stress in Past Societies. Peter Lang, Bern, 325-50.

Devolder D (2004) Générations démographiques et générations familiales. Temporalités. Revue de Sociologie, 1, 16-28.

Gaymu, J., Busque, M. A., Légaré, J., Décarie, Y., Vézina, S., \& Keefe, J. (2010). What will the family composition of older persons be like tomorrow? A comparison of Canada and France. Canadian Journal on Aging, 29(1), 57-71. https://doi.org/10.1017/S0714980809990419

Gaymu, J., Ekamper, P., \& Beets, G. (2007). Qui prendra en charge les Européens âgés dépendants en 2030 ? Population, 62(4), 789. https://doi.org/10.3917/popu.704.0789

Gilbert, J. P., \& Hammel, E. A. (1966). Computer Simulation and Analysis of Problems in Kinship and Social Structure. American Anthropologist, 68(1), 71-93. https://doi.org/10.1525/aa.1966.68.1.02a00070

Goodman, L. A., Keyfitz, N., \& Pullum, T. W. (1975). Addendum to Family formation and the frequency of various kinship relationships. Theoretical Population Biology, 8(3), 376381. https://doi.org/10.1016/0040-5809(75)90053-2

Goodman, Leo A., Keyfitz, N., \& Pullum, T. W. (1974). Family formation and the frequency of various kinship relationships. Theoretical Population Biology, 5(1), 1-27. https://doi.org/10.1016/0040-5809(74)90049-5 
Hammel, E. A. (1990). Socsim II. A Sociodemographic Microsimulation Program Rev. 1.0 Operating Manual. Berkeley: University of California.

Keyfitz, N., \& Caswell, H. (2005). Applied Mathematical Demography. Third Edition. New York: Springer.

Laslett, P. (1965). The World We Have Lost. London: Methuen.

Laslett, P. (1988). La parenté en chiffres. Annales. Economies, Société, Civilisations, 43(1), 524.

Le Bras, H. (1973). Parents, grands-parents, bisaïeux. Population, 28(1), 9-38.

Le Bras, H. (1982). Evolution des liens de famille au cours de l'existence. In Ined (Ed.), Les âges de la vie. Actes du colloque national de démographie (Vol. 1, pp. 27-45). Paris: INED Presses Universitaires de France.

Léridon, H. (2004). Can assisted reproduction technology compensate for the natural decline in fertility with age? A model assessment. Human Reproduction, 19(7), 1548-1553.

Léridon, H. (2008). A new estimate of permanent sterility by age: sterility defined as the inability to conceive. Population Studies, 62(1), 15-24. https://doi.org/10.1080/00324720701804207

Lesthaeghe, R. J., \& Page, H. J. (1980). The Post-Partum Non-Susceptible Period: Development and Application of Model Schedules. Population Studies, 34(1), 143-169.

Li, J., \& O'Donoghue, C. (2013). A survey of dynamic microsimulation models: uses, model structure and methodology. International Journal of Microsimulation, 6(2), 3-55. https://doi.org/10.34196/ijm.00082

Lotka, A. (1931). Orphanhood in relation to demographic factors. Metron, IX, 37-109.

Ma, Y., Shen, Z., \& Nguyen, D. T. (2016). Agent-based simulation to inform planning strategies for welfare facilities for the elderly: Day care center development in a Japanese city. Jasss, 19(4). https://doi.org/10.18564/jasss.3090

Mason, C. (2016). Socsim Oversimplified. Berkeley.

Morand, E., Toulemon, L., Pennec, S., Baggio, R., \& Billari, F. (2010). Demographic modelling : the state of the art (No. 2.1a). Paris. 
Nepal, B., Brown, L., Kelly, S., \& Percival, R. (2011). Projecting the Need for Formal and Informal Aged Care in Australia: A dynamic microsimulation approach. Academia.Edu, (June). Retrieved from http://utah.natsem.canberra.edu.au/storage/WP7 Final.pdf

Noble, J., Silverman, E., Rossiter, S., Evandrou, M., Bullock, S., Vlachantoni, A., \& Falkingham, J. (2012). Linked Lives: the utility of an agent-based approach to modeling partnership and household formation in the context of social care. Proceedings of the 2012 Winter Simulation Conference. https://doi.org/10.1109/WSC.2012.6465264

Preston, S. H. (1976). Family sizes of children and family sizes of women. Demography, 13(1), 105-114. https://doi.org/10.2307/2060423

Rodriguez, G., \& Trussel, J. (1980). Maximum Likelihood Estimation of the Parameters of Coale's Model Nuptiality Schedule from Survey Data. Word Fertility Survey, Technical Bulletins, $N^{\circ} 7$.

Ruggles, S. (1987). Prolonged Connections: The Rise of the Extended Family in Nineteenthcentury England and America. Maddison: University of Wisconsin Press.

Silverman, E., Bijak, J., Noble, J., Cao, V., \& Hilton, J. (2014). Semi-Artificial Models of Populations: Connecting Demography with Agent-Based Modelling. Advances in Computational Social Science, (November), 177-189. https://doi.org/10.1007/978-4431-54847-8_12

Smith, J. E. (1987). The computer simulation of kin sets and kin counts. In J. Bongaarts, T. Burch, \& K. Wachter (Eds.), Family Demography. Methods and their Applications (pp. 228-248). Oxford: Clarendon Press.

Smith, J. E., \& Oeppen, J. (1993). Estimating numbers of kin in historical England using demographic microsimulation. In D. Reher \& R. Schofied (Eds.), Old and New Methods in Historical Demography (pp. 280-317). Oxford: Clarendon Press.

Spielauer, M. (2007). Dynamic Microsimulation of Health Care Demand, Health Care Finance and the Economic Impact of Health Behavior: Survey and Review. International Journal of Microsimulation, 1(1), 35-53.

Spijker, J., Devolder, D., \& Zueras, P. (2020). The impact of demographic change in the balance between formal and informal old-age care in Spain. Results from a mixed 
microsimulation-agent-based model. Ageing and Society, 1-26. https://doi.org/10.1017/S0144686X20001026

Toulemon, L. (1995). The place of children in the history of couples. Population, an English Selection, 7, 163-186.

van Imhoff, E. (2005). LIPRO 4.0 Tutorial.

Wachter, K. W., Blackwell, D., \& Hammel, E. A. (1997). Testing the validity of kinship microsimulation. Mathematical and Computer Modelling, 26(6), 89-104. https://doi.org/10.1016/S0895-7177(97)00172-6

Wachter, Kenneth W, Hammel, E. A., \& Laslett, P. (1978). Statistical Studies of Historical Social Structure. New York: Academic Press.

Willekens, F. (2005). Description of the micro-simulation model. In Work (Vol. 8).

Willekens, F. (2010). Family and household demography. In Y. Zeng (Ed.), Encyclopedia of Life Support Systems. Vol II. Demography (pp. 86-112). Oxford: UNESCO in partnership with EOLSS Publishers.

Zagheni, E. (2015). Microsimulation in Demographic Research. In J. D. Wright (Ed.), International Encyclopedia of the Social \& Behavioral Sciences, Vol. 15 (2nd Editio, pp. 343-346). https://doi.org/10.1016/b0-08-043076-7/02115-x

Zhao, Z. (2006). Computer microsimulation and historical study of social structure : A comparative review of SOCSIM and CAMSIM. Revista de Demografía Histórica, XXIV(II), 59-88. 